\title{
Squeezing light into subwavelength metallic tapers: single mode matching method
}

\author{
Qiao Min and Reuven Gordon \\ Department of Electrical and Computer Engineering, University of Victoria, 3800 Finnerty \\ Rd, Victoria, B.C., V8P 5C2, Canada \\ rgordon@uvic.ca
}

\begin{abstract}
A simple single mode matching (SMM) method is proposed to analyze the optical throughput for subwavelength metal tapers. Both a 2D gold-clad taper gap and a 3D gold taper rod are analyzed with the SMM method. Including metal losses, these structures are calculated to have optimal squeezing taper angles of $14^{\circ}$ with $71 \%$ efficiency and $32^{\circ}$ with $44 \%$ efficiency, which is in good quantitative agreement with previous finite-difference timedomain simulations. The SMM method uses only analytic calculations and 2-by-2 matrix multiplication. Therefore, compared to past approaches, the SMM method is an efficient method to optimize the subwavelength metallic taper structures, while including losses, and it can be extended to more complex tapers and gratings in an obvious way. It also has the potential for fully analytic calculations.
\end{abstract}

Keywords: Surface plasmons, surface waves, subwavelength structures.

\section{INTRODUCTION}

Surface plasmon localization and enhancement in nano-strucutures has drawn great interest for its potential use in near-field optical microscopy and lithography [1-4], subwavelength optical waveguiding [5-8], and surface enhanced Raman scattering, which allows singlemolecule detection [9-14]. To efficiently coupling light into nanoscale devices, many structures have been designed [15-18]. As one solution, the subwavelength metallic taper structure allows for significant field enhancement towards the end of the taper [19-21]. Since the coupling efficiency of taper structure is dependent on the taper angle, optimizations to these structures have considered solving for the optimal angle. Two methods have been reported to calculate the optimal angle of the taper structures: the finite-difference timedomain method (FDTD) and the adiabatic theory [20, 22, 23]. FDTD is a comprehensive fully-vectorial method that can achieve accurate results; however, plasmonic structures require small grid sizes, and for stability, small time-steps. Therefore, FDTD is computationally intensive and time-consuming, which is a limitation in large structures in 2D, such as a gradual taper, and 3D structures. The adiabatic method is fully-analytic and efficient, but only accurate when the taper structure satisfies adiabaticity. Further calculations are required to ensure that there is negligible reflection.

In this paper, we propose a simple single mode matching (SMM) method that allows for rapid calculation of the optical transmission efficiency through both $2 \mathrm{D}$ and $3 \mathrm{D}$ taper structures. This method calculates the reflection and transmission of the lowest order mode at a stepped-interface, including the influence of material loss in each segment. By comparison with past results, it is verified that the SMM method can efficiently achieve accurate results under different configurations, including both loss and reflection. Examples of a 2D gold taper gap and a 3D gold taper rod are presented, which have been calculated in the past by FDTD and the adiabatic method $[22,23]$. Our results compare well quantitatively with those works. The proposed method can be easily extended to more complex structures. 


\section{THE SMM METHOD IN 2D}

\subsection{Structure and Gap Mode Description}

To demonstrate the SMM method, we analyze in detail a tapered gap in 2D. The analyzed structure is shown in Fig. 1. All the configurations are exactly the same as previous work for comparison [22]. A vacuum taper gap is formed between two gold media. The initial width of the taper, $W_{i}$ is $316.4 \mathrm{~nm}$, and the final width, $W_{f}$ is $1.512 \mathrm{~nm}$. The relative permittivity for the gold $\varepsilon_{m}$ is set to $-16.2+0.5 \mathrm{i}$, and for vacuum $\varepsilon_{v}$ is 1 . This structure is uniform and infinite in $z$-direction.

The wave mode in the taper gap used to calculate the optical transmission efficiency is the lowest order TM surface plasmon (SP) mode, which has the transverse magnetic field component in $z$-direction and electric field component in $x$ - and $y$-directions. The wavelength in vacuum $\lambda_{v}$ is $632.8 \mathrm{~nm}$. Only the $y$-component electric field and $z$ component magnetic field are considered, because they are the transverse components required for formulating the orthogonality relations.

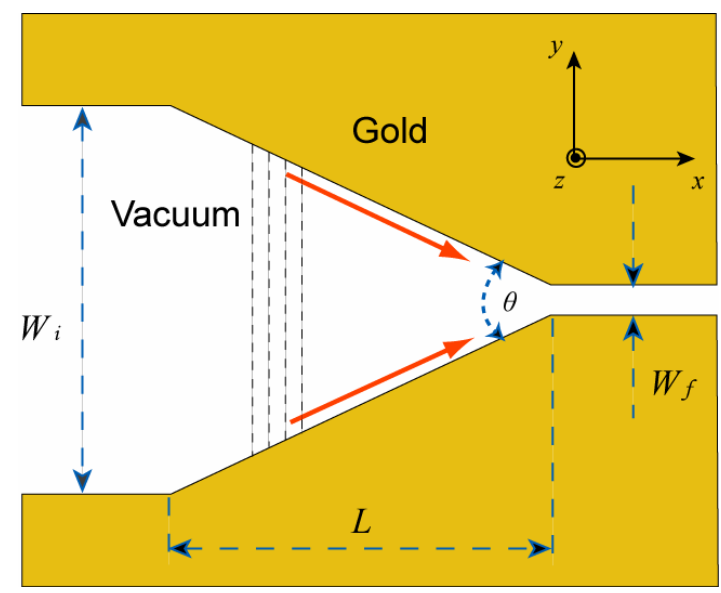

Fig. 1. The geometry of a 2D taper gap structure between two gold media. The widths $W_{i}$ and $W_{f}$ are fixed and the length $L$ varies for different taper angle $\theta$. For comparison with past works, $W_{i}=316.4 \mathrm{~nm}, W_{f}=1.512 \mathrm{~nm}$ and the permittivity of gold $\varepsilon_{m}$ is $-16.2+0.5 \mathrm{i}, \varepsilon_{v}$ is 1 for vacuum.

\subsection{Method}

The methodology here is based on the mode matching theory truncated to a single mode [24]. The taper gap is divided into small steps along $x$-direction (as shown in Fig. 2), and the method is then implemented on each two adjacent steps to calculate the transmission matrix $M_{t}$. A $0.1 \mathrm{~nm}$ step size is applied here.

Assuming two adjacent steps, step 1 and step 2, in which

$$
\left\{\begin{array}{l}
E_{1}^{y}=A_{1} E_{1 i}^{y}+B_{1} E_{1 r}^{y} \\
H_{1}^{z}=A_{1} H_{1 i}^{z}-B_{1} H_{1 r}^{z}
\end{array}\right.
$$




$$
\left\{\begin{array}{l}
E_{2}{ }^{y}=A_{2} E_{2 i}^{y}+B_{2} E_{2 r}^{y} \\
H_{2}{ }^{z}=A_{2} H_{2 i}^{z}-B_{2} H_{2 r}^{z}
\end{array} .\right.
$$

$E_{1}{ }^{y}, E_{2}{ }^{y}, H_{1}{ }^{z}, H_{2}{ }^{z}$ represent $y$-component electric field and $z$-component magnetic field in each step, respectively. Subscript $i$ and $r$ indicate the incident wave and the reflected wave. $A_{1}, A_{2}, B_{1}, B_{2}$ are the elements of transmission matrix $\mathrm{M}_{\mathrm{t}}$,

$$
\mathrm{M}_{\mathrm{t}}=\left[\begin{array}{ll}
A_{1} & B_{1} \\
A_{2} & B_{2}
\end{array}\right],
$$

and can be solved using Eqs. (1) and (2) and the orthogonality relations:

$$
\left\{\begin{array}{l}
\int_{-\infty}^{\infty}\left(E_{1}{ }^{y} \times H_{1}{ }^{z}\right) d y=\int_{-\infty}^{\infty}\left(E_{2}{ }^{y} \times H_{1}{ }^{z}\right) d y \\
\int_{-\infty}^{\infty}\left(E_{2}{ }^{y} \times H_{1}{ }^{z}\right) d y=\int_{-\infty}^{\infty}\left(E_{2}{ }^{y} \times H_{2}{ }^{z}\right) d y
\end{array} .\right.
$$

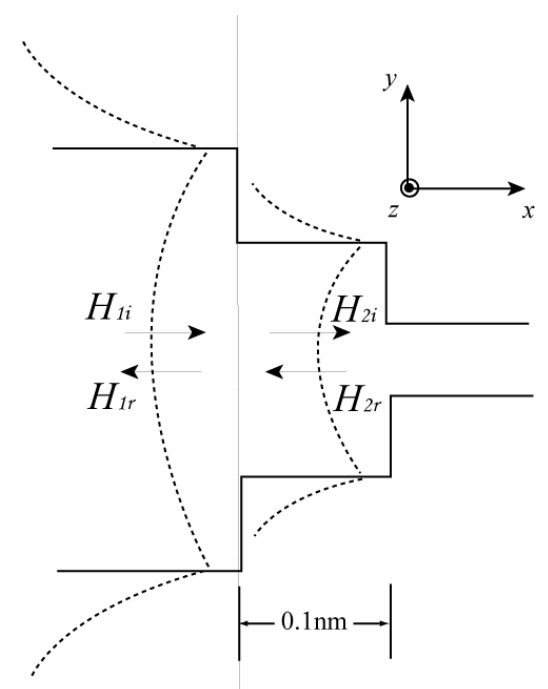

Fig. 2. The schematic diagram of the SMM model. The calculation step size in $x$-direction is $0.1 \mathrm{~nm} . H_{i}$ and $H_{r}$ represent the incident and reflective transverse magnetic fields, respectively.

To take into account the influence of the dissipation in each step, the propagation matrix $\mathrm{M}_{\mathrm{p}}$ should be considered, which is formed by

$$
\mathrm{M}_{\mathrm{p}}=\left[\begin{array}{cc}
e^{-j \beta \Delta x} & 0 \\
0 & e^{j \beta \Delta x}
\end{array}\right] .
$$

$\beta$ is the SP wave vector, and $\Delta x$ is the distance SP propagating in a step. 
The transmission efficiency result can be achieved by

$$
T_{\text {total }}=\prod_{\alpha}\left(\mathrm{M}_{\mathrm{p} \alpha} \cdot \mathrm{M}_{\mathrm{t} \alpha}\right) \cdot\left[\begin{array}{l}
1 \\
0
\end{array}\right] .
$$

Subscript $\alpha$ indicates the step number. For this 2D taper gap structure, the magnetic and electric fields can be expressed by the function of the gap width $w$,

$$
\begin{gathered}
H^{z}= \begin{cases}C_{1} \cdot \cosh \left(-\sqrt{\beta^{2}-\varepsilon_{v} k_{v}{ }^{2}} \cdot y\right) \cdot e^{-j \beta x} & |y| \leq \frac{w}{2} \\
C_{2} \cdot \exp \left(-\sqrt{\beta^{2}-\varepsilon_{m} k_{v}^{2}} \cdot\left(|y|-\frac{w}{2}\right)\right) \cdot e^{-j \beta x} & |y|>\frac{w}{2}\end{cases} \\
E^{y}= \begin{cases}-\frac{\beta}{\omega \varepsilon_{v}} H^{z} & |y| \leq \frac{w}{2} \\
-\frac{\beta}{\omega \varepsilon_{v} \varepsilon_{m}} H^{z} & |y|>\frac{w}{2}\end{cases}
\end{gathered}
$$

where $C_{1}$ and $C_{2}$ are the normalization coefficients, $\omega$ is the angular frequency of SP wave and $k_{v}$ is the wave vector in vacuum. The wave vector in the taper gap $\beta$ is also a function of $w$ and can be determined by equation [25],

$$
\tanh \left(\sqrt{\beta^{2}-k_{v}^{2}} \cdot \frac{w}{2}\right)=\frac{-\varepsilon_{v} \sqrt{\beta^{2}-\varepsilon_{m} k_{v}^{2}}}{\varepsilon_{m} \sqrt{\beta^{2}-\varepsilon_{v} k_{v}^{2}}}
$$

\subsection{Results}

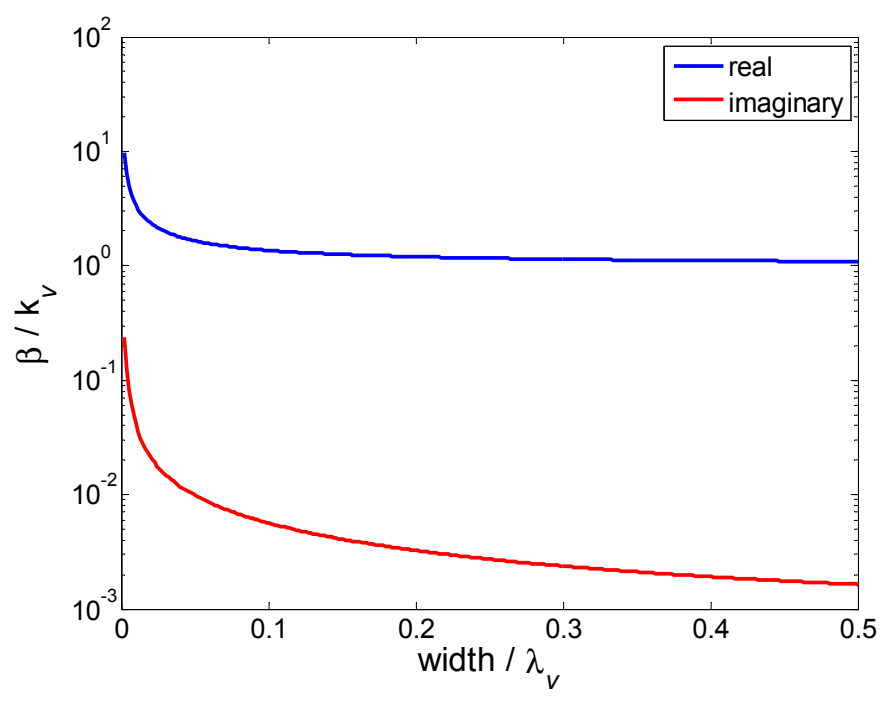

Fig. 3. The SP wave vector $\beta$ at different widths in the taper gap. Blue line represents the real part of $\beta$, and red line is the imaginary part. 
Figure 3 shows the dependence of the wave vector $\beta$ on the gap width. The real part (blue line) of $\beta$ is close to vacuum wave vector $k_{v}$ when the gap width $w$ is above $0.3 \lambda_{v}$, and then becomes large as $w$ decreases. Similar to the real part, the imaginary part (red line) of $\beta$ is very small $\left(\sim 0.001 k_{v}\right)$ when $w$ is $0.5 \lambda_{v}$ and increase rapidly when $w$ close to 0 . For the imaginary part of wave vector is related to the dissipation, it can be seen that the wave loss is significant with small $w$ even the permittivity imaginary part of the gold is only 0.5 in this example.

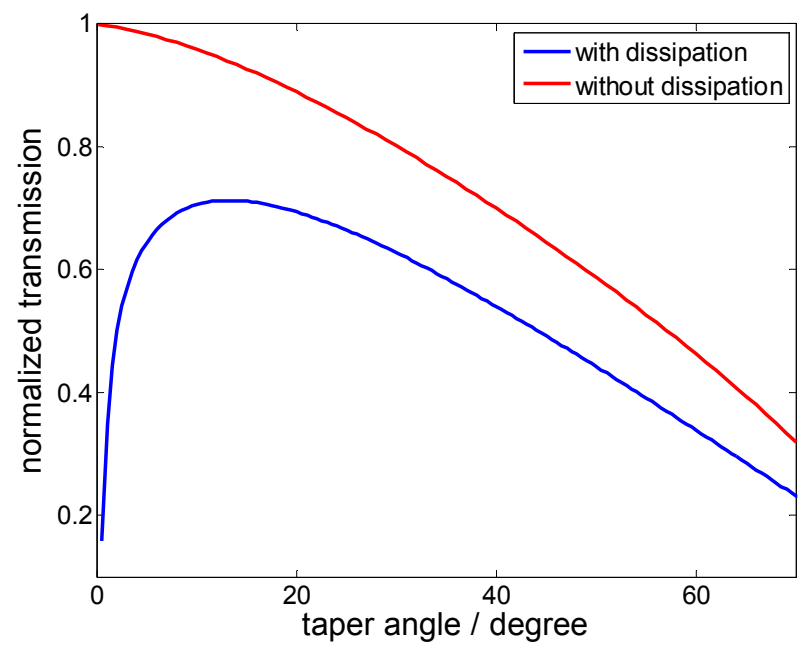

Fig. 4. The dependence of the normalized optical transmission efficiency through the taper gap on the taper angle $\theta$. Blue line: with dissipation, in which $\varepsilon_{m}=-16.2+0.5$; Red line: without dissipation, $\varepsilon_{m}=-16.2$.

Figure 4 shows the dependence of the normalized optical transmission efficiency through the taper gap structure on the taper angle $\theta$, calculated using Eqs. (1)-(9). Both the cases with dissipation and without dissipation are calculated. For the case without dissipation, the imaginary part of the relative permittivity is set to zero. Without dissipation (red line), the transmission efficiency is unity when $\theta$ is zero because there is negligible back-scattering for this adiabatic taper. The transmission efficiency decreases as the taper angle becomes larger due to backscattering. With the dissipation (blue line), the transmission efficiency goes to zero for $0^{\circ}$, since longer tapers have greater material losses. The transmission efficiency increases to maximum about 0.71 when $\theta$ is $14^{\circ}$. This is the optimum angle for the trade-off between back-scattering that dominates for large angles and losses that dominate for small angles. For the limit situation, the whole structure is equivalent to an infinite 2D subwavelength waveguide at $0^{\circ}$ taper angle which is corresponding to infinite dissipation and a gold plate with a $1.512 \mathrm{~nm}$ wide slit at $180^{\circ}$ which is corresponding to maximum reflection. This optimum angle for the 2D taper gap agrees well with the previous work using comprehensive FDTD calculations where the value of $13.5^{\circ}$ was found as the optimum [22].

For consistency within this work, the optical transmission efficiency of a $2 \mathrm{D}$ taper gap with another gold permittivity $\left(\varepsilon_{m}\right.$ is $\left.-11.44+1.12 \mathrm{i}\right)$ has also been investigated [23, 26], which is shown in Fig. 5. It can be seen that this taper gap structure has larger transmission efficiency at high taper angle condition than the former one due to its smaller reflection, but lower transmission peak $\left(0.58\right.$ at $\left.22.5^{\circ}\right)$ due to loss. 


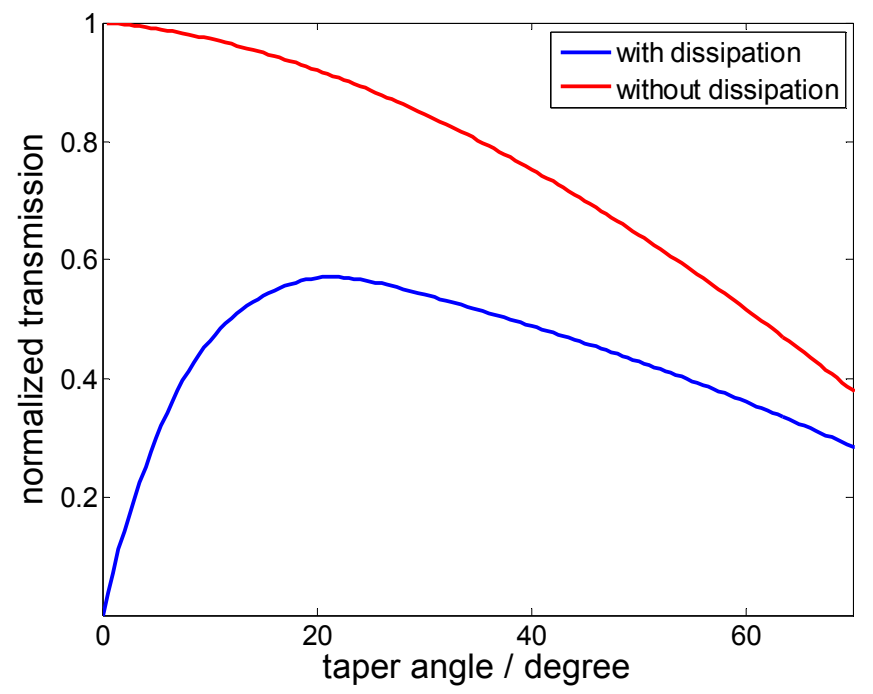

Fig. 5. The dependence of the normalized optical transmission efficiency through the taper gap on the taper angle $\theta$. Blue line: with dissipation, in which $\varepsilon_{m}=-11.44+1.12 \mathrm{i}$; Red line: without dissipation, $\varepsilon_{m}=-11.44$.

To further test the validity of the SMM method, a 3D gold taper rod is considered below.

\section{THE SMM METHOD IN 3D}

\subsection{Structure and Gap Mode Description}

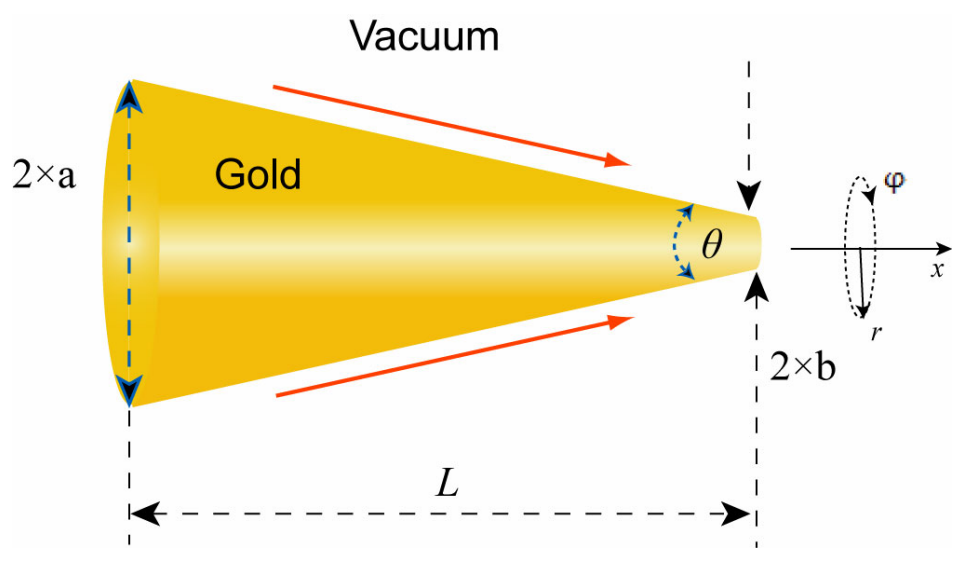

Fig. 6. The model of a $3 \mathrm{D}$ gold taper rod structure in vacuum. Here $2 \times a=$ $600 \mathrm{~nm}, 2 \times b=10 \mathrm{~nm}$ and the permittivity of gold $\varepsilon_{m}$ is $-11.44+1.12 \mathrm{i}, \varepsilon_{v}$ is 1 for vacuum.

Figure 6 shows the model of the 3D gold taper rod structure in vacuum. The vacuum wavelength $\lambda_{v}$ is $632.8 \mathrm{~nm}$. The initial diameter $2 \times a$ and the final diameter $2 \times b$ are $600 \mathrm{~nm}$, 
$10 \mathrm{~nm}$, respectively. The gold permittivity $\varepsilon_{m}$ is $-11.44+1.12 \mathrm{i}$, and the vacuum permittivity $\varepsilon_{v}$ is 1 . (Note that the different value of the relative permittivity here is used for direct comparison with past numerical calculations).

The SP wave mode used here is the lowest order surface plasmon TM mode, whose magnetic field is parallel to the azimuthal direction in the cylindrical coordinate system. Therefore, the $\varphi$-component of magnetic field and the $r$-component of electric field are considered for the orthogonality relations. The vacuum wavelength $\lambda_{v}$ of SP wave is also $632.8 \mathrm{~nm}$. All the configurations are the same as a recent work [23].

Figure 7 shows the schematic diagram of the SMM mode in 3D gold taper rod. The calculation step in $x$-direction is $0.1 \mathrm{~nm}$ as shown.

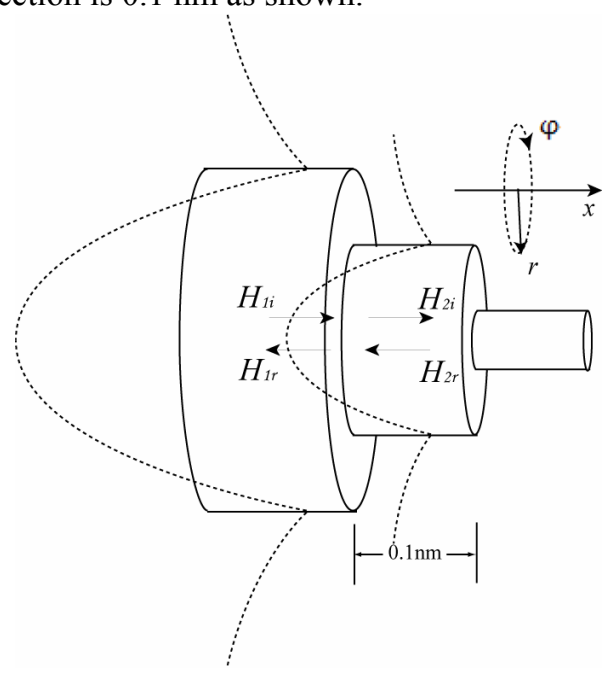

Fig. 7. The schematic diagram of the SMM model in 3D gold rod. The calculation step size in $x$-direction is $0.1 \mathrm{~nm} . H_{i}$ and $H_{r}$ represent the incident and reflective transverse magnetic fields, respectively.

\subsection{Method}

A similar methodology is applied in this example as in the 2D case. Instead of $E^{y}, H^{z}$, the $r$-component electric field $E^{r}$ and $\varphi$-component magnetic field $H^{\varphi}$ are considered:

$$
H^{\varphi}=\left\{\begin{array}{lc}
C_{1} \frac{j \omega \varepsilon_{m}}{p_{m}} I_{1}\left(p_{m} r\right) & r \leq \rho \\
-C_{2} \frac{j \omega \varepsilon_{v}}{p_{v}} K_{1}\left(p_{v} r\right) & r>\rho
\end{array},\right.
$$

and

$$
E^{r}=\left\{\begin{array}{ll}
-\frac{\beta}{\omega \varepsilon_{m}} H^{\varphi} & r \leq \rho \\
-\frac{\beta}{\omega \varepsilon_{v}} H^{\varphi} & r>\rho
\end{array} .\right.
$$


$C_{1}, C_{2}$ are the normalization coefficients, $I$ and $K$ are the first kind and the second kind of modified Bessel functions, respectively, and the subscripts indicate the orders [27]. $\omega$ is the angular frequency of SP wave and $\rho$ is the rod radius at certain position. $p$ is defined as

$$
\left\{\begin{array}{l}
p_{v}{ }^{2}=\beta^{2}-k_{v}{ }^{2} \\
p_{m}{ }^{2}=\beta^{2}-k_{m}{ }^{2}
\end{array} .\right.
$$

$k_{v}, k_{m}$ are the wave vectors in vacuum and gold, respectively. Using the boundary condition, SP wave vector $\beta$ can be achieved by equation below [27],

$$
\frac{K_{0}\left(p_{m} \rho\right)}{I_{0}\left(p_{v} \rho\right)}+\frac{\varepsilon_{m} p_{v}}{\varepsilon_{v} p_{m}} \frac{K_{1}\left(p_{m} \rho\right)}{I_{1}\left(p_{v} \rho\right)}=0
$$

Here the orthogonality relations are in form as:

$$
\left\{\begin{array}{l}
\int_{0}^{2 \pi} d \varphi \int_{0}^{\infty}\left(E_{1}^{r} \times H_{1}{ }^{\varphi}\right) r d r=\int_{0}^{2 \pi} d \varphi \int_{-\infty}^{\infty}\left(E_{2}{ }^{r} \times H_{1}{ }^{\varphi}\right) r d r \\
\int_{0}^{2 \pi} d \varphi \int_{-\infty}^{\infty}\left(E_{2}{ }^{r} \times H_{1}{ }^{\varphi}\right) r d r=\int_{0}^{2 \pi} d \varphi \int_{-\infty}^{\infty}\left(E_{2}{ }^{r} \times H_{2}{ }^{\varphi}\right) r d r
\end{array} .\right.
$$

\subsection{Results}

Figure 8 shows the dependence of the wave vector $\beta$ on the diameter $2 \rho$. The real part (blue line) of $\beta$ is close to $k_{v}$ when diameter is over 0.2 wavelength but increases rapidly when the diameter is made very small. The imaginary part (red line) is $0.01 k_{v}$ when the diameter equals to half $\lambda_{v}$ and increases rapidly to $k_{v}$ when the taper rod diameter decreases to $0.01 \lambda_{v}$.

Figure 9 shows the dependence of the normalized optical transmission efficiency through the gold taper rod structure on the taper angle $\theta$, as calculated using the same basic method as in Eqs. 1-5, but with modification to the cylindrical geometry as represented by Eqs. 10-14. Without dissipation, the transmission efficiency decreases from 1 at zero degree to 0.42 at $\theta$ $=60^{\circ}$. For there is lossless-metal, mode reflection in this case is larger than the $2 \mathrm{D}$ taper gap case, which contributes to the larger optimal taper angle. With dissipation (blue line), the optical transmission efficiency reaches a maximum of 0.44 at $\theta=32^{\circ}$. As is consistent with the $2 \mathrm{D}$ case, the transmission is zero at $0^{\circ}$ due to material losses over an infinitely long taper. Therefore, the optimal taper angle is bigger than in $2 \mathrm{D}$, which is due to the combined effects of greater loss and greater mode reflection. 


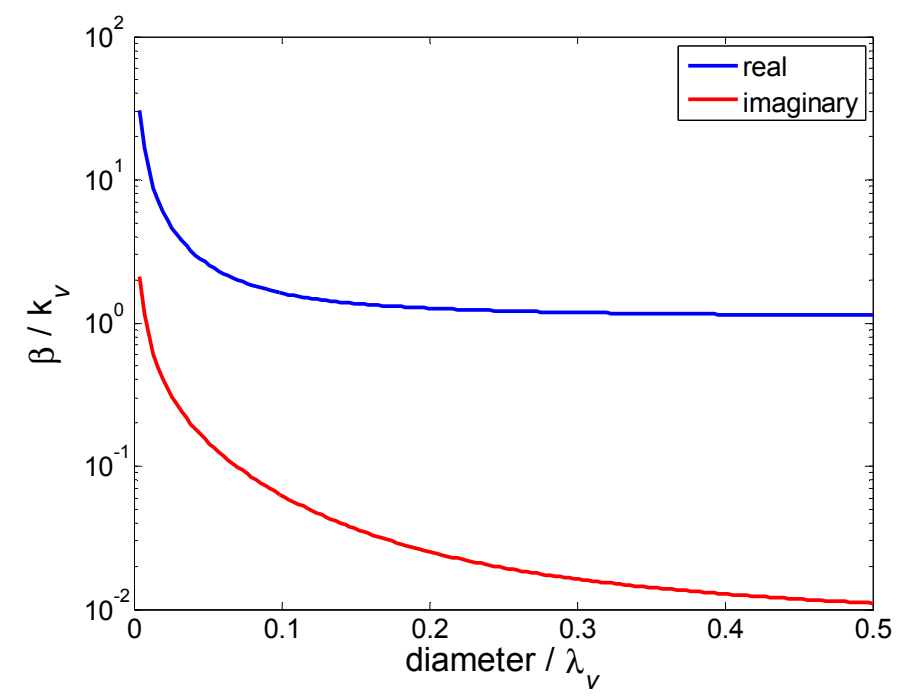

Fig. 8. The SP wave vector $\beta$ at different widths in the taper gap. Blue line represents the real part of $\beta$, and red line is the imaginary part.

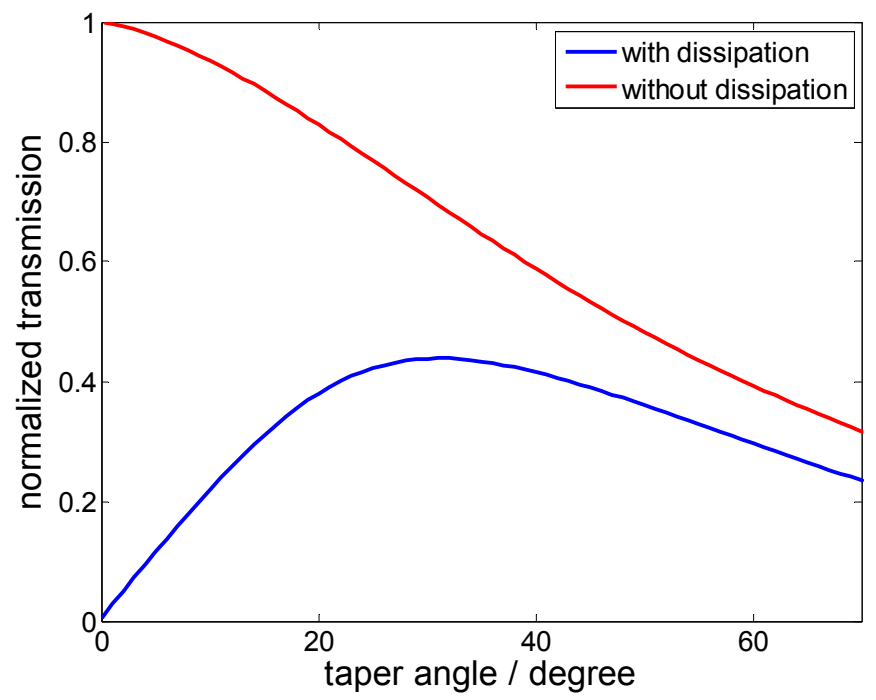

Fig. 9. The dependence of the normalized optical transmission efficiency through the gold taper rod on the taper angle $\theta$. Blue line: with dissipation, in which $\varepsilon_{m}=-11.44+1.12 \mathrm{i}$; Red line: without dissipation, $\varepsilon_{m}=-11.44$.

In a recent work, the adiabatic method has been applied to small taper angle realm and the FDTD method to large taper angle realm. The optimal taper angle for maximum electric field at exit side was $35^{\circ}$, which is in good agreement with the result of this paper [23]. 


\section{DISCUSSION}

\subsection{Comparison with Other Methods}

Two other methods have been used to calculate the SP wave propagation properties through metal taper structures: the FDTD method and the adiabatic method. Both of them have very distinct advantages and drawbacks as discussed above. Here we add the SMM method. The SMM method introduced by this paper considers both the mode reflection and dissipation, which allows for operation over a wide range of angles (approximately $0^{\circ}-70^{\circ}$, as discussed below). This method inherently contains the physics associated with the lowest-order mode, so that it is easy to interpret the results in terms of the properties of that mode, and to make generalizations to other geometries. Furthermore, the method is very efficient due to its analytic nature.

\subsection{Validity of the SMM Method}

Considering past FDTD calculations, there is discrepancies with the SMM work for angles greater than $90^{\circ}$ [22]. In particular, the FDTD calculations show a faster reduction in the transmitted light than the SMM method. This error is due to the truncation of the SMM method to a single mode only, whereas there is scattering to higher order modes for highlyangled tapers. While in principle, this problem can be solved by adding higher modes into the calculation, this will remove the benefit of simplicity in the proposed approach. Therefore, we suggest that this method may be applied for tapers in the range of approximately $0^{\circ}-70^{\circ}$, and for larger angles, comprehensive numerical methods should be used. Neglecting higher-order modes also removes the possibility of coupling to anti-symmetric modes in systems where there is symmetry breaking.

\subsection{Extensions of this Work}

The SMM method described here can be generalized to other structures, not only simple linear tapers. For example, it is possible to consider a nonlinear taper structure that would reduce the back scattering and enhance the transmission efficiency. For the 3D gold taper rod, at the optimum angle $32^{\circ}, 28 \%$ of the transmission loss is from back scattering, which means there is room for further the enhancement in the transmission efficiency by optimizing the taper geometry with a nonlinear taper.

Another possible extension of this work is the application of the SMM method to periodic structures. These structures are interesting for potential Bragg resonances [28, 29]. This would require using periodic conditions (or scaled periodic conditions when including losses) in the matrix calculations.

Finally, an interesting extension of this work would be to deduce analytic results for the optical transmission based on the SMM method. Since the method is semi-analytic in the present form, a parametric description of the mode-matching and propagation would allow for a fully-analytic treatment. While that proposal is a challenging endeavor, beyond the scope of this present investigation, it has the potential benefit of allowing for a fully analytic determination of the optimum structure simply by taking a derivative.

\section{CONCLUSION}

In summary, a simple SMM method is presented to calculate the optical transmission efficiency through subwavelength metal taper structures. It is found that a $14^{\circ}$ optimal angle with $71 \%$ transmission efficiency for the 2D taper gap structure in gold and a $32^{\circ}$ optimal angle with $44 \%$ transmission efficiency for the $3 \mathrm{D}$ taper gold rod structure, which both agree well with the previous results $[22,23]$. The method is more efficient than comprehensive 
numerical calculations and more accurate than adiabatic methods; it retains the influence of reflection and losses simply by multiplying out 2-by-2 matrices. The SMM method may be extended to other nonlinear geometries, for example, to optimize the subwavelength squeezing of light or to enhance Bragg resonances. Many applications that presently use plasmonic field enhancements stand to benefit from the SMM method, such as SERS which allows the technique to be sensitive enough to detect single molecule [9-14].

\section{References}

[1] E. Betzig and R. J. Chichester, "Single molecules observed by near-field scanning optical microscopy," Science 262, 1422-1425 (1993) [doi:10.1126/science.262.5138.1422].

[2] E. Betzig, J. K. Trautman, T. D. Harris, J. S. Weiner, and R. L. Kostelak, "Breaking the diffraction barrier - optical microscopy on a nanometric scale," Science 251, 1468-1470 (1991) [doi:10.1126/science.251.5000.1468].

[3] W. Srituravanich, N. Fang, C. Sun, Q. Luo, and X. Zhang, "Plasmonic nanolithography," Nano Lett. 4, 1085-1088 (2004) [doi:10.1021/n1049573q].

[4] M. M. Alkaisi, R. J. Blaikie, S. J. McNab, R. Cheung, and D. R. S. Cumming, "Subdiffraction-limited patterning using evanescent near-field optical lithography," Appl. Phys. Lett. 75, 3560-3562 (1999) [doi:10.1063/1.125388].

[5] S. I. Bozhevolnyi, V. S. Volkov, E. Devaux, and T. W. Ebbesen, "Channel plasmonpolariton guiding by subwavelength metal grooves," Phys. Rev. Lett. 95, 046802 (2005) [doi:10.1103/PhysRevLett.95.046802].

[6] S. I. Bozhevolnyi, V. S. Volkov, E. Devaux, J.-Y. Laluet, and T. W. Ebbesen, "Channel plasmon subwavelength waveguide components including interferometers and ring resonators," Nature 440, 508-511 (2006) [doi:10.1038/nature04594].

[7] E. Moreno, F. J. Garcia-Vidal, S. G. Rodrigo, L. Martin-Moreno, and S. I. Bozhevolnyi, "Channel plasmon-polaritons: modal shape, dispersion, and losses," Opt. Lett. 31, 3447-3449 (2006) [doi:10.1364/OL.31.003447].

[8] D. F. P. Pile and D. K. Gramotnev, "Channel plasmon-polariton in a triangular groove on a metal surface," Opt. Lett. 29, 1069-1071 (2004) [doi:10.1364/OL.29.001069].

[9] A. Campion and P. Kambhampati, "Surface-enhanced Raman scattering," Chem. Soc. Rev. 27, 241-250 (1998) [doi:10.1039/a827241z].

[10] K. Kneipp, Y. Wang, R. R. Dasari, and M. S. Feld, "Approach to single-molecule detection using surface-enhanced resonance Raman-scattering (serrs) - a study using rhodamine 6g on colloidal silver," Appl. Spectrosc. 49, 780-784 (1995) [doi:10.1366/0003702953964480].

[11] K. Kneipp, Y. Wang, H. Kneipp, L. T. Perelman, I. Itskan, R. R. Dasari, and M. S. Feld, "Single molecule detection using surface-enhanced Raman scattering (SERS)," Phys. Rev. Lett. 78, 1667-1670 (1997) [doi:10.1103/PhysRevLett.78.1667].

[12] S. M. Nie and S. R. Emery, "Probing single molecules and single nanoparticles by surface-enhanced Raman scattering," Science 275, 1102-1106 (1997) [doi:10.1126/science.275.5303.1102].

[13] Z. Q. Tian, B. Ren and D.Y. Wu, "Surface-enhanced Raman scattering: From noble to transition metals and from rough surfaces to ordered nanostructures," J. Phys. Chem. B 106, 9463-9483 (2002) [doi:10.1021/jp0257449].

[14] H. X. Xu, E. J. Bjerneld, M. Kall, and L. Borjesson, "Spectroscopy of single hemoglobin molecules by surface enhanced Raman scattering," Phys. Rev. Lett. 83, 4357-4360 (1999) [doi:10.1103/PhysRevLett.83.4357]. 
[15] M. W. Knight, N. K. Grady, R. Bardhan, F. Hao, P. Nordlander, and N. J. Halas, "Nanoparticle-mediated coupling of light into a nanowire," Nano Lett. 7, 2346-2350 (2007) [doi:10.1021/n1071001t].

[16] A. Hartschuh, H. N. Pedrosa, L. Novotny, and T. D. Krauss "Simultaneous fluorescence and Raman scattering from single carbon nanotubes," Science 301, 1354-1356 (2003) [doi:10.1126/science.1087118].

[17] S. J. Oldenburg, G. D. Hale, J. B. Jackson, and N. J. Halas, "Light scattering from dipole and quadrupole nanoshell antennas," Appl. Phys. Lett. 75, 1063-1065 (1999) [doi:10.1063/1.124597].

[18] B. Wang and G. P. Wang, "Surface plasmon polariton propagation in nanoscale metal gap waveguides," Opt. Lett. 29, 1992-1994 (2004) [doi:10.1364/OL.29.001992].

[19] A. Rasmussen and V. Deckert, "New dimension in nano-imaging: breaking through the diffraction limit with scanning near-field optical microscopy," Anal. Bioanal. Chem. 381, 165-172 (2005) [doi:10.1007/s00216-004-2896-3].

[20] M. I. Stockman, "Nanofocusing of optical energy in tapered plasmonic waveguides," Phys. Rev. Lett. 93, 137404 (2004) [doi:10.1103/PhysRevLett.93.137404].

[21] D. P. Tsai, A. Othonos, and M. Moskovits, "Raman-spectroscopy using a fiber optic probe with subwavelength aperture," Appl. Phys. Lett. 64, 1768-1770 (1994) [doi:10.1063/1.111802].

[22] D. F. P. Pile and D. K. Gramotnev, "Adiabatic and nonadiabatic nanofocusing of plasmons by tapered gap plasmon waveguides," Appl. Phys. Lett. 89, 041111 (2006) [doi:10.1063/1.2236219].

[23] D. K. Gramotnev, M. W. Vogel, and M. I Stockman, "Optimized nonadiabatic nanofocusing of plasmons by tapered metal rods," J. Appl. Phys. 104, 034311 (2008) [doi:10.1063/1.2963699].

[24] D. M. Pozar, Microwave Engineering, $3^{\text {rd }}$ ed., Wiley, Hoboken, NJ (2004).

[25] R. Gordon, "Light in a subwavelength slit in a metal: Propagation and reflection," Phys. Rev. B. 73, 153405 (2006) [doi:10.1103/PhysRevB.73.153405].

[26] P. B. Johnson and R. W. Christy, "Optical constants of the noble metals," Phys. Rev. B 6, 4370 (1972) [doi:10.1103/PhysRevB.6.4370].

[27] C. Yeh and F. Shimabukuro, The Essence of Dielectric Waveguides, Springer, New York (2008) [doi: 10.1007/978-0-387-49799-0].

[28] Q. Liu, L.-L. Wang, M.-D. He, W.-Q. Huang, D. Wang, B. S. Zou, and S. Wen, "A wide bandgap plasmonic Bragg reflector," Opt. Exp. 16, 4888-4894 (2008) [doi:10.1364/OE.16.004888].

[29] J. Park, H. Kim, and B. Lee, "High order plasmonic Bragg reflection in the metalinsulator-metal waveguide Bragg grating," Opt. Exp. 16, 413-425 (2008) [doi:10.1364/OE.16.000413]. 